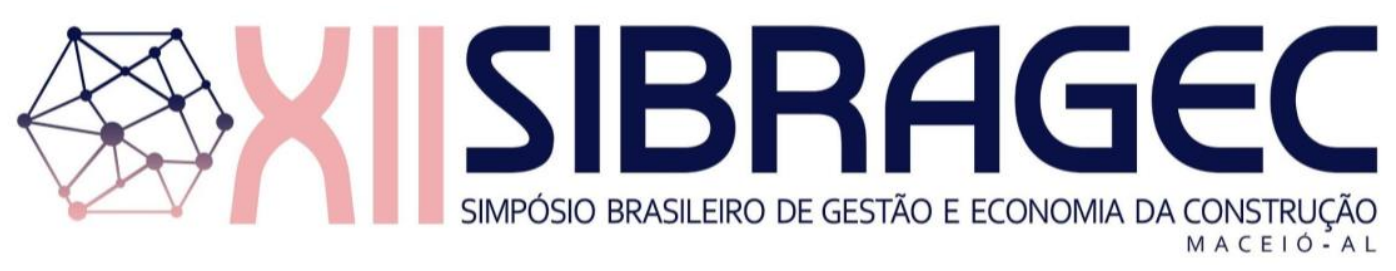

\title{
AVALIAÇÃO DE ATENDIMENTO A REQUISITOS DE DESEMPENHO NO PROJETO A PARTIR DO ARQUIVO IFC ${ }^{1}$
}

\author{
NICOLAI, Paola Oliveira de (1); SOUZA, Flávia Rodrigues de (2); \\ (1) Universidade de São Paulo, paola.nicolai@usp.br (2) Universidade de São Paulo, \\ flavia.souza@usp.br
}

\begin{abstract}
RESUMO
As construções residenciais brasileiras, desde 2013, precisam atender os critérios lançados pela NBR 15.575, que impactaram o processo de projeto significativamente. O Building Information Modelling (BIM) trouxe novos processos e ferramentas que estão ajudando os profissionais a melhorarem os seus projetos, mas na maioria das vezes, a inspeção dos modelos ainda é feita manualmente. Desta forma, o objetivo deste artigo é propor uma solução para avaliação de requisitos de desempenho de projetos residenciais a partir da extração de dados do formato IFC, utilizando códigos de programação em Python, bem como validar esta ferramenta com profissionais da construção civil. Para alcançar esse objetivo, um critério da norma técnica foi escolhido e os dados necessários inseridos em um modelo criado pelos autores, para serem verificados com a parametrização das regras no ambiente de programação. Além disso, realizou-se uma sessão de grupo focal com coordenadores de projetos para discutir essa abordagem. Os resultados demonstraram que a ferramenta tem grande potencial para a verificação de um certo grupo de critérios de desempenho, sendo reconhecida como uma solução que pode agregar valor ao processo, mesmo que haja algumas considerações importantes relacionadas ao perfil dos coordenadores e aos projetistas do setor.
\end{abstract}

Palavras-chave: NBR 15.575, IFC, Modelagem da Informação da Construção.

\begin{abstract}
Brazilians' residential constructions, since 2013, need to meet requirements launched by NBR15.575, that significantly impacted the design process. Building Information Modelling (BIM) has brought new processes and tools that are helping professionals to improve their design but, in most of the times, model inspection for code compliance is still done manually. Thus, this article aims to propose a solution for evaluating performance requirements of residential building projects through data extraction of IFC format using Python programing language, as to validate this tool with professionals of construction industry. To achieve this goal, one requirements of the standard was chosen, and the required data was created in a model elaborated by the authors to be verified with the rule's parametrization in the programming environment. Besides that, a focus group was conducted with project managers to discuss this approach. The results demonstrated that the tool has a great potential to check a specific group of performance requirements, being recognized as a solution that can aggregates great value to the process, even with some important considerations regarding managers profiles and designers of the sector.
\end{abstract}

Keywords: NBR 15.575, IFC, Building Information Modelling.

\section{INTRODUÇÃ̃O}

Entre os diferentes tipos de requisitos que guiam a elaboração de projetos no setor da Arquitetura, Engenharia e Construção, destacam-se aqueles advindos de códigos

\footnotetext{
${ }^{1} \mathrm{NICOLAI}$, P. O. de; SOUZA, F. R. de. Avaliação de atendimento a requisitos de desempenho no projeto a partir do arquivo IFC. In: SIMPÓSIO BRASILEIRO DE GESTÃO E ECONOMIA DA CONSTRUÇÃO, 12., 2021, Maceió. Anais[...] Porto Alegre: ANTAC, 2021. p.1-8. Disponível em: https://eventos.antac.org.br/index.php/sibragec/article/view/521. Acesso em: 2 out. 2021.
} 
regulatórios e normas técnicas, que são estabelecidos para garantir funções mínimas das construções, bem como a segurança e necessidade de seus usuários. Neste contexto, o lançamento da ABNT NBR 15.575:2013 impactou significativamente o modo como os empreendimentos residenciais precisam ser concebidos, já que até então, as empresas não desenvolviam os seus produtos norteados pelo desempenho (OTERO, 2018).

A evolução do processo de projeto, principalmente com a adoção de novas tecnologias e metodologias como os do Building Information Modelling (BIM), vem auxiliando os profissionais do setor no maior atendimento aos requisitos de desempenho. Segundo Lobos e Trebilcock (2014), o BIM se diferencia de tecnologias CAD tradicionais principalmente pelo fato de a partir de um único modelo, conseguir gerar diferentes informações em um processo preciso e coordenado.

Embora o BIM não possa ser considerado algo novo e o seu uso ser crescente no Brasil, pouco ainda é explorado em relação às tecnologias de validação de dados em modelos. Em 2018, por exemplo, a Câmara Brasileira da Indústria da Construção Civil (CBIC) realizou uma pesquisa com o intuído de levantar os principais usos do BIM, em diferentes fases do ciclo de vida de empreendimentos, no setor AEC do país. Os resultados indicaram que existe uma lacuna no domínio tecnológico pelos profissionais do setor da construção brasileira, uma vez que apenas 3\% dos respondentes apontaram a utilização da validação de códigos em suas atividades. Nesse contexto, Preidel e Borrmann (2016) afirmam que a inspeção manual de projetos é muitas vezes trabalhosa e propensa a erros. Reforçando essa ideia, Lee et al. (2016) relatam que a verificação manual de múltiplos requisitos em um modelo digital consome muito tempo, resultando em uma checagem incompleta e arbitrária.

Desta forma, o objetivo do artigo é apresentar uma proposta para a extração de características relacionadas a critérios da Norma de Desempenho de um arquivo no formato Industry Foundation Classes (IFC), a partir de programação em Python, de maneira a avaliar se o projeto está de acordo ou não com esses requisitos. Também é objetivo deste artigo apresentar a percepção de profissionais do setor da construção frente a esta mesma solução. O presente trabalho busca, assim, contribuir com a proposição de um sistema que possibilite a automatização da verificação de critérios de desempenho por parte da coordenação de projetos, também apresentando um diagnóstico da real viabilidade da sua aplicação na prática profissional atualmente.

A avaliação de sistemas para avaliar regras automáticas, principalmente relacionadas a códigos construtivos, vêm ganhando ampla discussão no meio acadêmico. Andrade e Arantes (2017), por exemplo, abordaram as potencialidades do software Solibri para a verificação de critérios da Norma de Desempenho. Contudo, as iniciativas que existem ainda estão muito centralizadas em softwares proprietários e pouco flexíveis aos usuários (LEE et al., 2016).

Nesse sentido, a utilização de formatos abertos, como IFC, para realizar a verificação de dados no modelo pode ser bastante vantajosa e prática. Segundo Luo e Gong (2014), um primeiro obstáculo para a verificação automática é que softwares BIM possuem os seus próprios formatos autorais e a utilização de API (Application Programming Interface) é limitado para este propósito. Desta forma, estes mesmos autores argumentam que o IFC é a única representação neutra e independente da construção, suportada pela maioria destas ferramentas.

O IFC veio para auxiliar a colaboração com o BIM e resolver questões de interoperabilidade entre diferentes softwares, trocando informações entre diferentes agentes independente de ferramentas autorais. O IFC é um esquema aberto criado pela BuildingSmart, tratando-se de uma biblioteca de dados que representam um 
empreendimento de construção, ao longo do seu ciclo de vida, caracterizando-se como um modelo extensível e orientado à objetos (EASTMAN et al., 2014).

É importante salientar que este trabalho está inserido em uma pesquisa em desenvolvimento pelos autores, em que se pretende propor um processo para coordenadores de projeto conseguirem melhor gerenciar requisitos de desempenho, acompanhado por diretrizes de verificação a partir da solução tecnológica proposta neste artigo.

\section{METODOLOGIA}

A construção da proposta teve como guia as recomendações feita por Eastman et al. (2009) para a elaboração de sistemas de checagem de informação: (1) interpretação da regra e posterior estruturação; (2) preparação do modelo da construção; (3) execução da verificação; (4) divulgação dos resultados.

É importante citar que antes mesmo da primeira etapa, foi realizada uma classificação prévia dos critérios de desempenho da ABNT NBR 15.575 para caracterizar os grupos de requisitos da norma, indicando-se os itens viáveis de serem analisados por meio do IFC. Embora o desenvolvimento do sistema de classificação não seja apresentado no artigo, os critérios que delimitam o presente estudo estão relacionados à especificação de projeto (materiais, equipamentos, etc.), bem como aqueles relacionados a características geométricas do modelo.

A Figura 1, a seguir, ilustra a estrutura da metodologia adotada, que será detalhada em sequência:

Figura 1 - Fluxo metodológico adotado

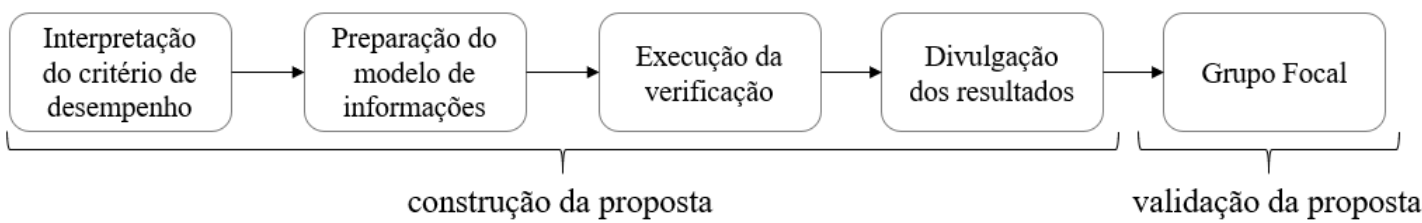

Fonte: AUTORES

(1) Interpretação da regra e posterior estruturação: para exemplificação da solução, será utilizado o seguinte critério: ABNT NBR 15.575 Parte 1 - 13.3.1 Níveis de iluminação artificial: "Os níveis gerais de iluminação promovidos nas diferentes dependências dos edifícios habitacionais por iluminação artificial devem atender ao disposto na Tabela 6 desta norma".

A escolha desse critério se justifica pelo fato do item estar dentro da delimitação proposta de classificação, isto é, trata-se de uma especificação de projeto. Vale ressaltar que não é a intenção deste estudo discutir um sistema de extração ou interpretação automática de códigos regulatórios, sendo essa parte feita manualmente. Desta forma, a regra é interpretada por meio da análise de um conjunto de parâmetros que caracterizam o respectivo critério (Quadro 1).

Adotou-se o sistema de classificação de objetos Omniclass como meio de aumentar a universalização da proposta. 
Quadro 1: Estudo dos parâmetros envolvidos para o critério exemplo

\begin{tabular}{|l|l|}
\hline Métrica de Referência & Lux \\
\hline Elementos do modelo & Espaços \\
\hline $\begin{array}{l}\text { Condições - Regra } \\
\text { *Nível Mínimo }\end{array}$ & $\begin{array}{l}\text { Sala de Estar; Dormitório; Banheiro; Área de Serviço > 100 } \\
\text { lux / Cozinha } 200 \text { lux } \\
\text { Corredores, Escadas, Garagens cobertas > 75 lux } \\
\text { Garagens descobertas > 20 lux }\end{array}$ \\
\hline $\begin{array}{l}\text { Sistema de classificação de } \\
\text { objetos - Omniclass }\end{array}$ & $\begin{array}{l}\text { Sala de Estar: 13-57 13 15 11 / Dormitório: } 13-651900 \\
\text { Banheiro: 13-23 17 00 / Área de Serviço: 13-65 17 } 00 \\
\text { Cozinha: 13-65 23 00 / Corredores: 13-25 11 11 } \\
\text { Escadas: 13-23 11 / Garagens cobertas: 13-21 13 00 } \\
\text { Garagens descobertas: 13-21 11 00 }\end{array}$ \\
\hline $\begin{array}{l}\text { Informação necessária no } \\
\text { modelo }\end{array}$ & $\begin{array}{l}\text { Parâmetro de projeto (Lux) conectado com o elemento } \\
\text { correspondente (espaço) }\end{array}$ \\
\hline
\end{tabular}

Fonte: AUTORES

(2) Preparação do modelo da construção: Um modelo teste de um edifício habitacional foi criado pelos autores no software Revit da Autodesk, versão 2020. Os espaços foram identificados conforme a classificação Omniclass correspondente. A informação necessária para a verificação foi criada, isso é, um parâmetro de projeto "Iluminação Artificial (lux)", onde valores aleatórios foram atribuídos para cada ambiente. Posteriormente, exportou-se o arquivo para o formado IFC com as configurações de "IFC 2x3 Coordination View 2.0 + Conjunto de Propriedades".

A versão ' $2 \times 3$ ' foi a escolhida por ainda ser a mais estável para o respectivo software de autoria em relação às versões mais novas.

(3) Execução da verificação: o ambiente de análise e criação dos códigos em Python foi o Jupyter Notebook, módulo utilizado através da ferramenta Anaconda Navigator. Foi utilizado a biblioteca IfcOpenShell (Open CASCADE) para viabilizar a exploração do arquivo IFC.

A criação dos códigos dependeu, além do conhecimento básico da linguagem de programação, do entendimento da estrutura do IFC para iniciar o processo e conseguir estruturar uma lógica de busca de informações dentro das entidades e respectivos atributos no arquivo. Nesse sentido, a consulta à documentação técnica lançada pela BuildingSmart foi fundamental.

É importante reforçar que a lógica adotada para o critério exemplo (Figura 2), não será necessariamente a mesma para a verificação dos demais itens da Norma de Desempenho que fazem parte da delimitação da pesquisa. Neste respectivo exemplo, a propriedade Iluminação Artificial (lux) está conectada aos espaços, mas há critérios que estão relacionados a outros elementos do modelo, a serem identificados pelo conjunto de parâmetros na realização da primeira etapa. 
Figura 2 - Entidades relacionadas ao parâmetro de Iluminação Artificial (lux)

Atributo: Related Objetcs

ifcSpace $\longleftarrow$ ifcreldefinesbyproperties $\longrightarrow$ ifcpropertyset $\longrightarrow \begin{aligned} & \text { ifcpropertysinglevalue } \\ & \text { name: lluminação Artificial (Lux) }\end{aligned}$

Fonte: AUTORES

(4) Divulgação dos resultados: As respostas foram obtidas no próprio ambiente dos códigos através dos status "Aprovado" e "Reprovado". Não foi dado nenhum tratamento de exportação ou comunicação posterior, como relatórios ou representações gráficas.

Embora só um critério de desempenho esteja sendo exemplificado no artigo, este processo se repetiu para todos os critérios selecionados como viáveis pela classificação citada no início da seção de metodologia.

A proposta então foi levada para uma sessão de Grupo Focal, digitalmente, envolvendo coordenadores de projeto do setor da construção civil e especialistas da Norma de Desempenho da região de São Paulo (Quadro 3). O objetivo da reunião do grupo foi validar a proposta e realizar um diagnóstico sobre a sua aplicabilidade na prática. Assim, a condução do grupo foi estruturada em três etapas, cujos objetivos e estratégias são apresentados, respectivamente, a seguir (Quadro 2) :

Quadro 2 - Objetivos e respectivas estratégias para cada etapa do grupo focal

\begin{tabular}{|l|l|l|}
\hline Etapa & Objetivo & Estratégia \\
\hline Etapa 1 & $\begin{array}{l}\text { Levantar o atual nível de } \\
\text { utilização do formato IFC } \\
\text { por coordenadores }\end{array}$ & $\begin{array}{l}\text { Alinhamento inicial de } \\
\text { conceitos }\end{array}$ \\
\hline Etapa 2 & $\begin{array}{l}\text { A avaliação dos } \\
\text { participantes em relação ao } \\
\text { sistema proposto }\end{array}$ & $\begin{array}{l}\text { Apresentação da solução } \\
\text { e demonstração, em } \\
\text { tempo real, da } \\
\text { verificação de alguns } \\
\text { critérios de desempenho }\end{array}$ \\
\hline Etapa 3 & $\begin{array}{l}\text { Levantar a percepção dos } \\
\text { participantes da } \\
\text { viabilidade e dificuldades } \\
\text { de utilização na prática da } \\
\text { solução }\end{array}$ & $\begin{array}{l}\text { Questionar o } \\
\text { conhecimento técnico da } \\
\text { coordenação em relação à } \\
\text { programação, resistências } \\
\text { e implantação }\end{array}$ \\
\hline
\end{tabular}

Fonte: AUTORES

Quadro 3 - Perfil dos participantes do grupo focal

\begin{tabular}{|l|l|l|l|}
\hline Participante & Formação & Atuação & Tipo de Empresa \\
\hline Participante A & Engenharia & $\begin{array}{l}\text { Consultoria da Norma de } \\
\text { Desempenho }\end{array}$ & Consultoria \\
\hline Participante B & Engenharia & Acadêmica & Universidade \\
\hline Participante C & Engenharia & Coordenação de Projetos & Gerenciadora \\
\hline Participante D & Arquitetura & Coordenação de Projetos & $\begin{array}{l}\text { Incorporadora e } \\
\text { Construtora }\end{array}$ \\
\hline
\end{tabular}

Fonte: AUTORES 


\section{RESULTADOS}

\subsection{Verificação de requisitos a partir do IFC}

O código, bem como o resultado para a verificação do critério exemplo, pode ser visto na Figura 3. A construção inicial das sentenças buscou identificar o parâmetro criado "Iluminação Artificial (lux)", através das entidades de propriedades. Em sequencia, solicitou-se ao programa a extração de todos os valores relacionados a este parâmetro e os respectivos espaços aos quais eles estão conectados. O passo seguinte foi a parametrização da regra, indicando o espaço correspondente com o valor de referência da norma.

Finalmente, foi solicitado ao programa imprimir o nome padrão do espaço, o valor do parâmetro atribuído no modelo e se estava "Aprovado" ou "Reprovado". Nesse caso, a garagem; a sala e a cozinha estão reprovadas. A escada de emergência, banheiro, corredor, área de serviço e quartos estão de acordo com a Norma de Desempenho.

\section{Figura 3 - Código para verificação do critério relacionado à Iluminação Artificial (lux)}

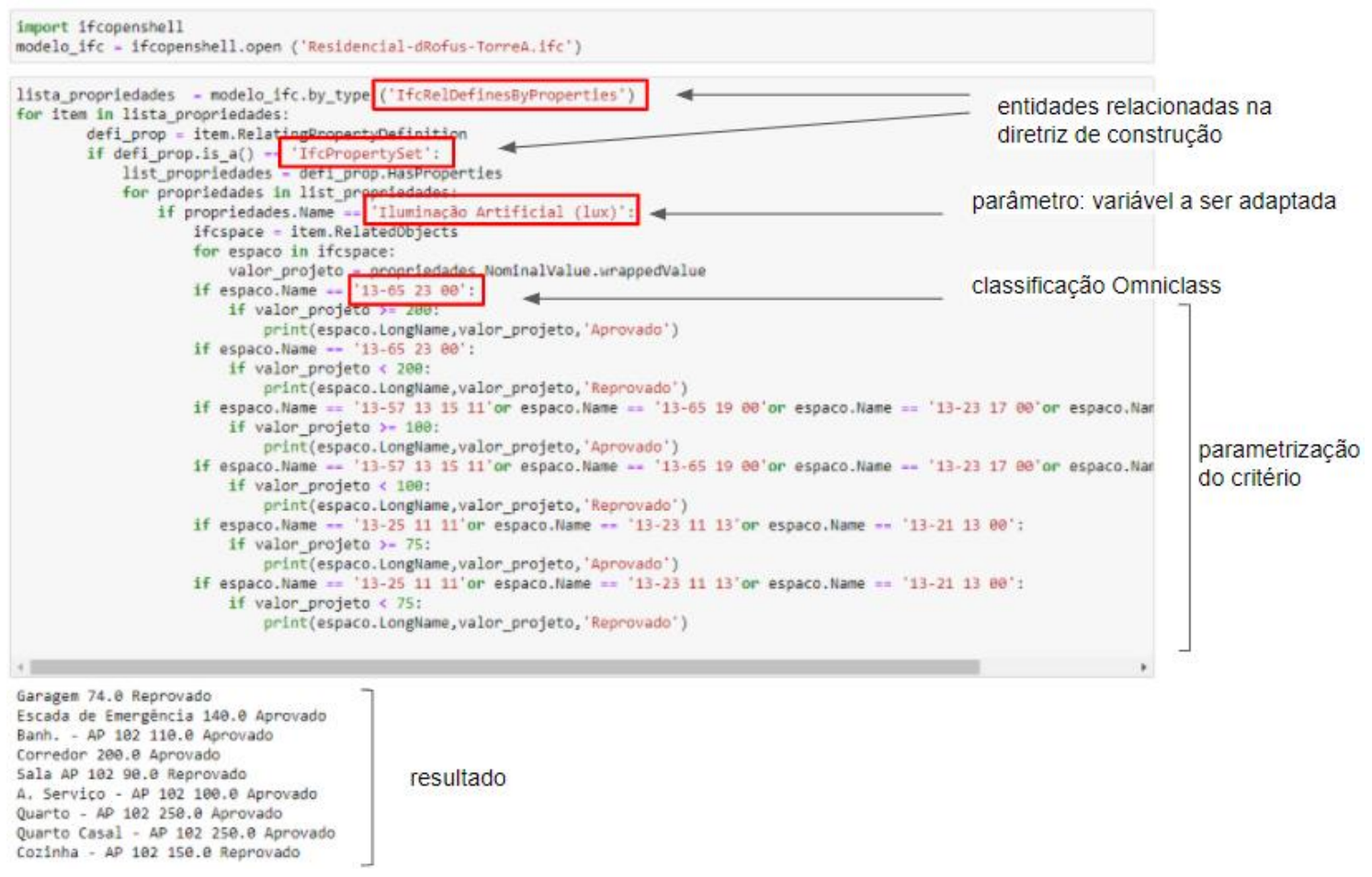

Fonte: AUTORES

\subsection{Sessão do Grupo Focal}

Como relatado na sessão de metodologia, a realização do Grupo Focal teve como objetivo validar a proposta anterior. A primeira etapa teve início e, após os alinhamentos de conceito, os participantes foram questionados sobre a atual utilização, por coordenadores de projeto, do IFC. Os especialistas entraram em consenso e acreditam que este formato já se faz presente para a maioria desses profissionais. Contudo, percebeu-se que todos os relatos apontaram que o uso do IFC estava limitado, principalmente, à visualização e análise do modelo de estrutura pela arquitetura, pelo primeiro ter origem em softwares mais específicos.

Na segunda etapa, após a demonstração da solução, a proposta foi bem aceita por todos os participantes, que afirmaram ser interessante, reforçando que mesmo que nem todos 
os critérios sejam possíveis de serem verificados dessa forma, a automatização é bemvinda para o que for possível.

Já iniciando a terceira etapa, o participante A expressou a preocupação de que para essa solução ser viável, as informações necessárias ao modelo precisam estar muito claras e detalhadas. Isso porque os projetistas estão acostumados a especificar de uma forma que não favorece a verificação de características técnicas dos produtos. Como exemplo, o participante A citou a indicação de um revestimento cerâmico de piso: é indicado somente a marca e a dimensão, desconsiderando-se características técnicas.

Em relação ao conhecimento técnico de coordenadores em relação à criação de códigos de programação, a reação ao questionamento foi de estranhamento e todos admitiram que esse conceito está bem distante de suas realidades. O participante D levantou a própria questão do perfil do coordenador de projetos, afirmando que hoje esse player precisa lidar com muitas atividades em paralelo, principalmente relacionadas à compatibilização e planejamento, o que acaba implicando na contratação de especialistas para casos mais técnicos.

O participante $\mathrm{C}$ afirmou que não sabe responder quais são as lacunas da proposta. Contudo, apontou que hoje haveria a necessidade de existir um coordenador BIM, pois como comentado, o coordenador de projetos não tem tempo ou até mesmo competências para lidar com as particularidades tecnológicas do BIM, como por exemplo programação em linguagem específica, por exemplo.

O participante A expressou que o caminho para chegar ao nível de competência requerida para os coordenadores, incluindo os conhecimentos para programação, é complexa. Ele relembrou que existe a lacuna do próprio conhecimento técnico em relação a Norma de Desempenho. Em relação às resistências, o mesmo participante também apontou o fato do atendimento dos requisitos ainda ser tratado em segundo plano e a baixa integração com os projetistas.

\section{CONSIDERAÇÕES FINAIS}

Em relação à proposta técnica de avaliação de requisitos de desempenho a partir da extração de dados de arquivos IFC, por meio de códigos de programação, pode-se considerar que o comportamento da solução, para os objetivos indicados, foi satisfatório. Além disso, as discussões do grupo focal validaram que a ferramenta traz contribuições para a automatização da verificação de requisitos.

Contudo, como limitações do uso dessa solução, como foi proposta, é importante reforçar, primeiramente, que está restrita a alguns itens da norma, isto é, quando estes estão relacionados à especificação de objetos ou parâmetros geométricos do modelo. Outro ponto relevante é que por mais que tenha se buscado utilizar elementos que universalizam os códigos para vários projetos, como a utilização da Omniclass, nem sempre é possível dentro das exigências de cada critério, o que indica que a proposta ainda exige considerável interação do usuário. Desta forma, de modo a viabilizar de fato a solução, o usuário precisa conhecer sobre os critérios de desempenho para saber as características necessárias que devem ser incluídas modelo; conhecer sobre a estrutura do IFC e como exportá-lo corretamente de softwares de autoria, além de conseguir identificar, pelo menos, onde estão as principais variáveis nos códigos de programação para possíveis ajustes.

Como contribuições secundárias, o artigo trouxe importantes reflexões sobre o atual perfil dos coordenadores de projeto e a interação com os projetistas, o que pode ser considerado para o desenvolvimento de outros trabalhos que tenham como recorte a gestão de projetos, a Norma de Desempenho e o BIM. Vale ressaltar que esse é um diagnóstico limitado a 
região de São Paulo. O debate realizado pelo grupo focal indica que o coordenador muitas vezes se afasta de um perfil mais técnico, atuando mais no controle de entregas do que na auditoria de projetos. Além disso, a atuação de projetistas na especificação das soluções de projeto precisa ser melhor discutido sob o enfoque das tecnologias BIM.

\section{REFERÊNCIAS}

ABNT. ASSOCIAÇÃO BRASILEIRA DE NORMAS TÉCNICAS. NBR $\mathbf{1 5 . 5 7 5}$ Edificações Habitacionais - Desempenho - Parte 1: Requisitos gerais. Rio de Janeiro: ABNT, 2013a.

ANDRADE, F.; ARANTES, E. Verificação automática de requisitos de projetos da norma de desempenho NBR 15.575 a partir da adequação de regras da plataforma BIM Solibri Model Checker. In: $1^{\circ}$ Simpósio Brasileiro de Tecnologia e Comunicação na Construção e $10^{\circ}$ Simpósio Brasileiro de Gestão e Economia da Construção. Fortaleza, Ceará, 2017.

CÂMARA BRASILEIRA DA INDÚSTRIA DA CONSTRUÇÃO. Road Show BIM Resultados da pesquisa e desdobramentos. Brasília: CBIC, 2018. Disponível em <https://cbic.org.br/inovacao/wp-content/uploads/sites/23/2018/05/RoadShow.pdf>.

Acesso em 11/10/2020

EASTMAN, C. et al. Manual de BIM: um guia de modelagem da informação da construção para arquitetos, engenheiros, gerentes, construtores e incorporadores. Bookman Editora, 2014.

EASTMAN, C. et al. Automatic rule-based checking of building designs. Automation in construction, vol. 18, n. 8, p. 1011-1033, Nov. 2009. DOI: 10.1016/j.autcon.2009.07.002

LEE, H.; LEE, J.; PARK, S.; KIM, I.Translating building legislation into a computerexecutable format for evaluating building permit requirements. Automation in Construction, v. 71, p. 49-61, Nov. 2016

LOBOS, D.; TREBILCOCK, M. Building performance information and graphs approach for the design of floor plans. ArquiteturaRevista, v. 10, n. 1, p. 23-30, Jan./Jun. 2014.

LUO, H.; GONG, P. A BIM-based Code Compliance Checking Process of Deep Foundation Construction Plans. Journal of Intelligent \& Robotic Systems, v. 79, p.549-576, 2014.

OTERO, J. A. Ferramenta de gestão de riscos baseada na teoria dos conjuntos Fuzzy para suporte à garantia do desempenho de edificações habitacionais.2018. 227f. Tese (Doutorado em Estruturas e Construção Civil)- Departamento de Engenharia Civil e Ambiental da Faculdade de Tecnologia da Universidade de Brasília, Brasília.

PREIDEL, C.; BORRMANN, A.Towards code compliance checking on the basis of a visual programming language. Journal of Information Technology in Construction, v.21, p. 402-421, Nov. 2016.

ZHANG, Chi et al. Interoperable validation for IFC building models using open standards. Journal of Information Technology in Construction, v. 20, p.24-39, jan. 2015.

ZHANG, J.; EL-GOHARY, N. M. Semantic NLP-based information extraction from construction regulatory documents for automated compliance checking. Journal of Computing in Civil Engineering, v. 30, n. 2, p. 04015014, 2016. 